\title{
SQUARES IN THE CENTRE OF THE GROUP ALGEBRA OF A SYMMETRIC GROUP
}

\author{
J. Murray* \\ Mathematics Department, \\ University College Dublin, \\ Belfield Dublin 4, Ireland.
}

Let $\mathcal{S}(n)$ be a finite symmetric group of degree $\mathrm{n}$ and let $F$ be a perfect field of characteristic $p>0$. We use $Z=Z(F \mathcal{S}(n))$ to denote the centre of the group algebra $F \mathcal{S}(n)$. If $\mathcal{X}$ is a subset of $\mathcal{S}(n)$ then $\mathcal{X}^{+}$denotes its sum in $F \mathcal{S}(n)$. As is well known $\left\{\mathcal{K}^{+} \mid \mathcal{K}\right.$ a conjugacy class of $\left.\mathcal{S}(n)\right\}$ forms an $F$-basis for $Z$. We use $Z_{p^{\prime}}$ to denote the $F$-subspace of $Z$ spanned by the $p$-regular class sums. The map $z \rightarrow z^{p}$ is a semi-linear transformation on $Z$, with respect to the automorphism $\lambda \rightarrow \lambda^{p}$ of $F$. Its image $Z^{p}$ is an $F$-subalgebra of $Z$, and its kernel $\left\{z \in Z \mid z^{p}=0\right\}$ is an ideal of $Z$. Our main result is:

Theorem 1. Let $p=2$. Then $Z^{2}=Z_{2^{\prime}}$. So $z \in Z$ is a square in $Z$ if and only if $z$ is an $F$-linear combination of 2-regular class sums.

A $p$-block of $\mathcal{S}(n)$ is an indecomposable $F$-algebra, which is a direct summand of $F \mathcal{S}(n)$. Each $p$-block $B$ of $\mathcal{S}(n)$ has an associated weight $w$ and $p$-core $\alpha$. So $w$ is an integer between 0 and $n / p$, while $\alpha$ is a partition of $n-w p$ which has no $p$-hooks. See [JK81, 2.7 and 6.1] for definitions and proofs. The number $k(B)$ of irreducible characters associated to $B$ equals the $F$-dimension of the centre $Z(B)$ of $B$, while the number $l(B)$ of irreducible Brauer characters equals the $F$-dimension of $Z_{2^{\prime}} \cap Z(B)$. Set $Z(B)^{2}=\left\{z^{2} \mid z \in Z(B)\right\}$. The following is a block version of Theorem 1 :

Theorem 2. Let $B$ be a 2-block of $\mathcal{S}(n)$, of weight $w$. Then $\operatorname{dim}\left(Z(B)^{2}\right)$ equals the number $P(w)$ of partitions of $w$.

Proof. We have $Z(B)^{2}=Z^{2} \cap Z(B)$, since $Z(B)$ is commutative and unital. So $Z(B)^{2}=Z_{2^{\prime}} \cap Z(B)$, by Theorem 1. But $\operatorname{dim}\left(Z_{2^{\prime}} \cap Z(B)\right)=l(B)=P(w)$, by $[\mathrm{O} 80,3.6]$. This proves the result.

Date: July 6, 2000.

1991 Mathematics Subject Classification. 20C20.

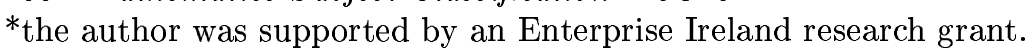


It seems unlikely that one could find an explicit formula for a square root of a 2-regular class sum (but see the proof of Proposition 9). We can at least show:

Theorem 3. Each 2-regular class of $\mathcal{S}(n)$ occurs with odd multiplicity in the square of some involution class.

In fact, for each 2-regular class of $\mathcal{S}(n)$, we can explicity describe a class of involutions for which this theorem holds. Our methods could be used to compute the square of any involution class sum of $\mathcal{S}(n)$.

For the rest of this paper we fix $g \in \mathcal{S}(n)$ and $D$ a Sylow $p$-subgroup of $\mathbf{C}_{\mathcal{S}(n)}(g)$, and set $\mathrm{C}=\mathbf{C}_{\mathcal{S}(n)}(D)$. We use $g_{p}\left(g_{p^{\prime}}\right)$ to denote the $p$-part ( $p$-regular part) of $g$. So $g_{p}$ has $p$-power order, $g_{p^{\prime}}$ has $p^{\prime}$-order and $g=g_{p} g_{p^{\prime}}=g_{p^{\prime}} g_{p}$.

Our notation for subgroups, centralizers and normalizers is standard.

Proposition 4. $\mathrm{C}=\left\langle g_{p}\right\rangle \times N$, for some group $N$.

We defer the proof of Proposition 4 to the end of the paper, and proceed immediately to the proof of two corollaries. Corollary 6 will be needed in the proof of Theorem 1, while Corollary 5 may be of independent interest.

Let $a \in F \mathcal{S}(n)$ and $x \in \mathcal{S}(n)$. We use $(a, x)$ to denote the coefficient of $x$ in $a$. Set $\Omega(x):=\left\{y \in \mathcal{S}(n) \mid y^{p}=x\right\}$. If $x$ has $p^{\prime}$-order, we use $x^{1 / p}$ to denote the unique element of $\Omega(x)$ that has $p^{\prime}$-order.

Corollary 5. $Z_{p^{\prime}}$ is a subalgebra of $Z$.

Proof. Let $\mathcal{K}$ and $\mathcal{L}$ be $p$-regular classes of $\mathcal{S}(n)$, and suppose that $g_{p} \neq 1_{\mathcal{S}(n)}$. It is enough to show that $\left(\mathcal{K}^{+} \mathcal{L}^{+}, g\right)=0$. Note that $g \notin N$, where $N$ is the normal subgroup of $\mathbf{C}_{\mathcal{S}(n)}(D)$ given by Proposition 4 . Now

$$
\begin{aligned}
\left(\mathcal{K}^{+} \mathcal{L}^{+}, g\right)= & \left((\mathrm{C} \cap \mathcal{K})^{+}(\mathrm{C} \cap \mathcal{L})^{+}, g\right), \\
& \quad \text { using the Brauer homomorphism, see }[\mathrm{K} 91,(54)], \\
= & 0, \quad \text { as } N \text { contains every 2-regular element of } \mathrm{C} .
\end{aligned}
$$

The corollary follows.

Let $m$ be a nonnegative integer. The proof of Corollary 5 actually shows that the $F$-subspace of $Z$ spanned by the class sums of elements of $\mathcal{S}(n)$ whose $p$-parts have order $p^{m}$ or less is a subalgebra of $Z$.

Corollary 6. $Z^{p} \subseteq Z_{p^{\prime}}$.

Proof. Let $\mathcal{K}$ be a conjugacy class of $\mathcal{S}(n)$, and suppose that $g_{p} \neq 1_{\mathcal{S}(n)}$. It is enough to show that $\left(\left(\mathcal{K}^{+}\right)^{p}, g\right)=0$. By $[K 91,(55)]$, we have

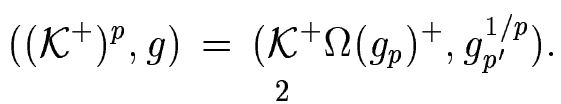


Now, $D$ acts by conjugation on $\mathcal{K}$ and $\Omega\left(g_{p}\right)$, and centralizes $g_{p^{\prime}}^{1 / p}$. Thus

$$
\left(\left(\mathcal{K}^{+}\right)^{p}, g\right)=\left((\mathrm{C} \cap \mathcal{K})^{+}\left(\mathrm{C} \cap \Omega\left(g_{p}\right)\right)^{+}, g_{p^{\prime}}^{1 / p}\right) .
$$

But Proposition 4 implies that $\mathrm{C} \cap \Omega\left(g_{p}\right)$ is empty. The result follows.

Corollary 6 implies the following, cf. [K91, (59)]:

Proposition 7. $\left\{z \in Z \mid z^{p}=0\right\}=\left\{z \in Z \mid z \Omega\left(1_{\mathcal{S}(n)}\right)^{+}=0\right\}$.

The analogues of Corollaries 5 and 6 hold for the alternating group $\mathcal{A}(n)$ also.

Proposition 8. $Z(F \mathcal{A}(n))_{p^{\prime}}$ is a subalgebra of $Z(F \mathcal{A}(n))$ and $Z(F \mathcal{A}(n))^{p} \subseteq$ $Z(F \mathcal{A}(n))_{p^{\prime}}$.

Proof. Suppose that $g$ is an element of $\mathcal{A}(n)$. If $p \neq 2$, then $D$ is a Sylow $p$-subgroup of $C \cap \mathcal{A}(n)=\mathbf{C}_{\mathcal{A}(n)}(g)$. In particular,

$$
\mathbf{C}_{\mathcal{A}(n)}(D)=\left\langle g_{p}\right\rangle \times M, \quad \text { for some group } M,
$$

using Proposition 4. Thus $Z(F \mathcal{A}(n))^{p} \subseteq Z(F \mathcal{A}(n))_{p^{\prime}}$ and $Z(F \mathcal{A}(n))_{p^{\prime}}$ is a subalgebra of $Z(F \mathcal{A}(n))$, exactly as in the proofs of Corollaries 5 and 6 .

Suppose now that $p=2$. Let $\mathcal{K}$ be a conjugacy class of $\mathcal{A}(n)$. Then either $\mathcal{K}$ is a conjugacy class of $\mathcal{S}(n)$, or the elements of $\mathcal{K}$ have cycle type $\alpha$, where $\alpha$ is a partition of $n$ into unequal odd parts (see [JK81, 1.2.10]). In the former case we have

$$
\left(\mathcal{K}^{+}\right)^{2} \in Z_{2^{\prime}} \cap Z(F \mathcal{A}(n))=Z(F \mathcal{A}(n))_{2^{\prime}},
$$

using Corollary 6. In the latter case $\mathcal{K}$ has 2-defect zero. It is a theorem of Brauer that the class sums of 2-defect zero classes span an ideal $Z_{0}$ of $Z(F \mathcal{A}(n))$. Since $Z_{0}$ is contained in $Z(F \mathcal{A}(n))_{2^{\prime}}$, it follows that $\left(\mathcal{K}^{+}\right)^{2} \in$ $Z(F \mathcal{A}(n))_{2^{\prime}}$ in this case also.

The proof that $Z(F \mathcal{A}(n))_{2^{\prime}}$ is a subalgebra of $Z(F \mathcal{A}(n))$ proceeds in a similar fashion.

Let $\mu=\left(\mu_{1}, \mu_{2}, \ldots, \mu_{t}\right)$ be a partition of $n$. So $\mu_{1}+\cdots+\mu_{t}=n$ and $\mu_{1} \geq$ $\mu_{2} \geq \cdots \geq \mu_{t}>0$. We use $|\mu|=t$ to denote the number of parts of $\mu$. The conjugacy classes of $\mathcal{S}(n)$ are parametrized by the partitions of $n$. The class corresponding to $\mu$ contains $\left(1, \ldots, \mu_{1}\right)\left(\mu_{1}+1, \ldots, \mu_{1}+\mu_{2}\right) \ldots\left(n-\mu_{t}+1, \ldots, n\right)$. Clearly this class is $p$-regular if and only if $\mu_{i}$ is coprime to $p$, for $i=1, \ldots, t$.

Let $K$ be an arbitrary integral domain. In [M83], G. E. Murphy defines elements $L_{u}$ in $K \mathcal{S}(n)$ by

$$
L_{u}:=(1, u)+(2, u)+\cdots+(u-1, u),
$$


where $u$ is any integer between 2 and $n$, and each $(v, u)$ is a transposition. For convenience, we set $L_{1}:=1_{\delta(n)}$.

Suppose that $1 \leq i<j<u$ or $u<i<j \leq n$. Then trivially $L_{u}(i, j)=$ $(i, j) L_{u}$. In particular

$$
L_{u} L_{v}=L_{v} L_{u}
$$

for all $u, v \in\{1, \ldots, n\}$. Also for $1 \leq u<n$, it can be shown that

$$
\begin{aligned}
L_{u} L_{u+1}(u, u+1) & =(u, u+1) L_{u} L_{u+1}, \quad \text { and } \\
\left(L_{u}+L_{u+1}\right)(u, u+1) & =(u, u+1)\left(L_{u}+L_{u+1}\right) .
\end{aligned}
$$

Now $1, L_{u} L_{u+1}$ and $L_{u}+L_{u+1}$ generate, as an algebra, the ring of symmetric polynomials in $L_{u}$ and $L_{u+1}$ over any commutative ring. It follows that the transposition $(u, u+1)$ commutes with any symmetric polynomial in $L_{u}$ and $L_{u+1}$. Since $\{(u, u+1) \mid 1 \leq u<n\}$ generate $\mathcal{S}(n)$, we conclude that any symmetric polynomial in $L_{2}, \ldots, L_{n}$ lies in the centre $Z(K \mathcal{S}(n))$ of $K \mathcal{S}(n)$.

Let $P(n, p)$ denote the number of partitions of $n$ into parts which are congruent to 1 modulo $p$.

Proposition 9. $\operatorname{dim}\left(Z^{p}\right) \geq P(n, p)$.

Proof. Let $\mu=\left(\mu_{1}, \mu_{2}, \ldots\right)$ be a partition of $n$. Suppose that $\mu_{i}>1$ for $i=1, \ldots, r$. Set $X^{\mu}$ as the sum, in $K \mathcal{S}(n)$, of all distinct products of the form

$$
\left(L_{u_{1}}\right)^{\mu_{1}-1}\left(L_{u_{2}}\right)^{\mu_{2}-1} \ldots\left(L_{u_{r}}\right)^{\mu_{r}-1}
$$

where $u_{1}, u_{2}, \ldots, u_{r}$ runs over all sets of $r$ elements from $2,3, \ldots, n$. If all parts of $\mu$ are 1 , then set $X^{\mu}:=1_{\mathfrak{S}(n)}$.

The main result of $[\mathrm{M} 83,1.9]$ is that if $g$ is an element of $\mathcal{S}(n)$ of cycle type $\mu$, then the coefficient of $g$ in $X^{\mu}$ is 1 , while if $\lambda=\left(\lambda_{1}, \lambda_{2}, \ldots\right)$ is the cycle type of any element of $\mathcal{S}(n)$ which occurs in $X^{\mu}$, then either $|\mu|<|\lambda|$ or $\mu \triangleleft \lambda$, where $\triangleleft$ is the dominance relation on partitions. Murphy uses these facts to show that $\left\{X^{\mu} \mid \mu\right.$ a partition of $\left.n\right\}$ forms a $K$-basis for $Z(K \mathcal{S}(n))$.

Now consider when $K=F$ is a field of characteristic $p$. Let $\mu$ be a partition of $n$ with $\mu_{i} \equiv 1(\bmod p)$, for $i=1, \ldots,|\mu|$. Set $\lambda_{i}=\left(\mu_{i}-1\right) / p+1$, for $i=1, \ldots,|\mu|$. Let $\lambda$ be the partition of $n$ whose first $|\mu|$ parts are $\lambda_{1}, \ldots, \lambda_{|\mu|}$, and whose remaining parts equal 1 . Using the fact that the $L_{u}$ commute, and the binomial theorem modulo $p$, we see that

$$
\left(X^{\lambda}\right)^{p}=X^{\mu} .
$$

The proposition now follows from the linear independence of the $X^{\mu}$.

We now give the proof of our main theorem. 
proof of Theorem 1. Clearly $P(n, 2)$ equals the number of 2-regular classes of $\mathcal{S}(n)$. So Proposition 9 implies that $\operatorname{dim}\left(Z^{2}\right) \geq \operatorname{dim}\left(Z_{2^{\prime}}\right)$. But $Z^{2} \subseteq Z_{2^{\prime}}$, by Corollary 6. The theorem follows.

A partition is called 2-singular if at least one of its parts is even.

Corollary 10. $\operatorname{dim}\left\{z \in Z \mid z^{2}=0\right\}$ equals the number of 2-singular partitions of $n$.

We need the following result on blocks of symmetric groups:

Proposition 11. Let $B$ be a p-block of $\mathcal{S}(n)$, of weight $w$. Then $Z(B) \cong$ $Z\left(B_{0}\right)$, where $B_{0}$ is the principal p-block of $\mathcal{S}(p w)$.

Proof. The principal $p$-block $B_{0}$ of $\mathcal{S}(p w)$ has empty core and weight $w$. M. Enguehard [E90] has shown that there exists a perfect isometry between any two $p$-blocks of finite symmetric groups that have the same weight. This implies, among other things, that the centres of $B$ and $B_{0}$ are isomorphic.

Let $B$ be a $p$-block of $\mathcal{S}(n)$, let $J(B)$ denote the Jacobson radical of $Z(B)$, and let $p^{t}$ denote the exponent of a defect group of $B$. Using Proposition 11, and (59) of [K91], we see that

$$
z^{p^{t}}=0, \quad \text { for each } z \in J(B) .
$$

This can be sharpened to:

Theorem 12. There exists $z \in J(B)$ with $z^{p^{t-1}} \neq 0$.

First we need two lemmas.

Let $G$ be a finite group. For each positive integer $m$, define

$$
\begin{aligned}
\Omega_{m} & :=\left\{x \in G \mid x^{p^{m}}=1_{G}\right\} \\
\Lambda_{m} & :=\left\{x \in G \mid o(x)=p^{m}\right\}=\Omega_{m} \backslash \Omega_{m-1} \\
\Delta_{m} & :=\left\{x \in G \mid x_{p} \in \Lambda_{m}\right\} .
\end{aligned}
$$

Lemma 13. Let e be an idempotent in $Z(F G)$. Then

$$
e \Lambda_{m}^{+}=\left(e, 1_{G}\right) \Lambda_{m}^{+}+\left(\text {terms involving non p-elements of } \Delta_{m}\right) \text {. }
$$

Proof. Let $x \in G$ have order $p^{m}$. It follows from a well-known result of Iizuka (see $[\mathrm{K} 91,(61)])$ that the support of $e \Lambda_{m}^{+}$is contained in $\Delta_{m}$. So it is enough 
to show that $\left(e \Lambda_{m}^{+}, x\right)=\left(e, 1_{G}\right)$. We have

$$
\begin{aligned}
\left(e \Lambda_{m}^{+}, x\right) & =\left(e \Omega_{m}^{+}, x\right)-\left(e \Omega_{m-1}^{+}, x\right) \\
& =\left(e^{p^{t}}, x^{p^{t}}\right)^{p^{-t}}-\left(e^{p^{t-1}}, x^{p^{t-1}}\right)^{p^{-t+1}}, \quad \text { by }(55) \text { of }[\mathrm{K} 91] \\
& =\left(e, 1_{G}\right)^{p^{-t}}-\left(e, x^{p^{t-1}}\right)^{p^{-t+1}} \\
& =\left(e, 1_{G}\right), \quad \text { as }\left(e, 1_{G}\right) \in G F(p) \text { and as } e \text { is supported } \\
& \text { on the } p \text {-regular elements of } G .
\end{aligned}
$$

Lemma 14. Let $c$ be an $m$-cycle, where $m \geq 2$, and let $t$ be a transposition that does not commute with $c$. Then tc is an $(m-1)$-cycle, an $(m+1)$-cycle, or a product of two commuting cycles whose combined length is $\mathrm{m}$.

Proof. This is a routine calculation.

Proof of Theorem 12. Let $e$ be the unique idempotent in $Z(B)$, and let $\omega$ denote the epimorphism $B \rightarrow F$ which has kernel $J(B)$. Using Proposition 11 , we may assume that $B$ is the principal $p$-block of $\mathcal{S}(n)$.

Let $\tau$ be the class of transpositions in $\mathcal{S}(n)$, and let $m$ be a positive integer. We may write

$$
\tau^{+} e=i+j
$$

where $i=\omega\left(\tau^{+}\right) e \in G F(p) e$ and $j \in J(B)$. If $m$ is a positive integer then

$$
\left(\tau^{+} e\right)^{p^{m}}=i^{p^{m}}+j^{p^{m}}=i+j^{p^{m}}
$$

So the proposition will follow if we show that $\left(\tau^{+} e\right)^{p^{t-1}} \neq\left(\tau^{+} e\right)^{p^{t}}$.

Let $u$ be a $\left(p^{t+1}\right)$-cycle in $\mathcal{S}(n)$. Then $u^{1 / p}$ is also a $\left(p^{t+1}\right)$-cycle. Using [K91, (55)], and the fact that $\left(\tau^{+} e \Omega_{m}^{+}, u\right) \in G F(p)$, we see that

$$
\left(\left(\tau^{+} e\right)^{p^{m}}, u\right)=\left(\tau^{+} e \Omega_{m}^{+}, u\right)
$$

It follows that

$$
\begin{aligned}
\left(\left(\tau^{+} e\right)^{p^{t}}, u\right)-\left(\left(\tau^{+} e\right)^{p^{t-1}}, u\right) & =\left(\tau^{+} e \Omega_{t}^{+}, u\right)-\left(\tau^{+} e \Omega_{t-1}^{+}, u\right) \\
& =\left(\tau^{+} e \Lambda_{t}^{+}, u\right) .
\end{aligned}
$$

Let $\lambda_{t}$ denote the class of $p^{t}$-cycles in $\mathcal{S}(n)$. Suppose that $t \in \tau$ and $x \in \Delta_{m}$ and $t x=u$. Then $x=t u$ contains a $p^{t}$-cycle in its cycle decomposition. So $x$ is a $p^{t}$-cycle, using Lemma 14. It then follows from Lemma 13 that

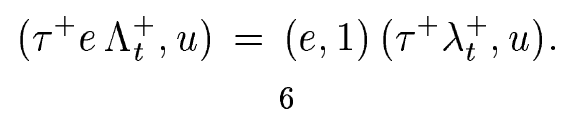


A direct calculation shows that

$$
\left|\left\{(t, l) \in \tau \times \lambda_{t} \mid t l=u\right\}\right|=p^{t}-1 .
$$

We conclude from (15), (16) and (17) that

$$
\left(\left(\tau^{+} e\right)^{p^{t}}, u\right)-\left(\left(\tau^{+} e\right)^{p^{t-1}}, u\right)=-(e, 1) .
$$

But $(e, 1) \neq 0_{F}$, by a theorem of Brauer. So $\left(\tau^{+} e\right)^{p^{t-1}} \neq\left(\tau^{+} e\right)^{p^{t}}$. This completes the proof.

Let $J(Z)$ denote the Jacobson radical of $Z$, and let $p^{t}$ denote the $p$-exponent of $\mathcal{S}(n)$. Suppose that $p=2$. If $n=4$ then $z^{p^{t-1}+1}=0$, for all $z \in J(Z)$, while if $n=6$, there exists $z \in J(Z)$ with $z^{p^{t}-1} \neq 0$. So Theorem 12 is best possible. On the other hand, the dihedral group $D_{8}$ of order 8 has 2-exponent 4 , yet $z^{2}=0$ for each $z \in J\left(Z\left(F D_{8}\right)\right)$. So Theorem 12 does not generalize to all finite groups.

Corollary 18. Let $J(Z)$ denote the Jacobson radical of $Z$, and let $p^{t}$ denote the p-exponent of $\mathcal{S}(n)$. Then $\operatorname{dim}_{F}\left(J(Z)^{p^{t-1}}\right)$ is greater that or equal to the number of $p$-blocks of $\mathcal{S}(n)$ that have weight greater than or equal to $p^{t-1}$.

Proof. Suppose that $B$ is a $p$-block of $\mathcal{S}(n)$, of weight $w \geq p^{t-1}$. Now by [JK81, 6.2.39], a defect group $D$ of $B$ is isomorphic to a wreath product of a cyclic group of order $p$ and a Sylow $p$-subgroup of a Symmetric group of degree $w$. But $w<p^{t}$. So the $p$-adic decomposition of $w$ contains $p^{t-1}$ with non-zero multiplicity. It follows that $D$ has a direct factor isomorphic to a Sylow $p$ subgroup of $\mathcal{S}\left(p^{t}\right)$. Hence $D$ has exponent $p^{t}$. The corollary now follows from Theorem 12.

Theorem 19. Let $p$ be an odd prime. Then $Z^{p} \lesseqgtr Z_{p^{\prime}}$.

Proof. Let $\tau$ be the class of transpositions in $\mathcal{S}(n)$. So $\tau^{+} \in Z_{p^{\prime}}$. Suppose that there exists $z \in Z$ with $z^{p}=\tau^{+}$. Then $z^{p^{t}}=\left(\tau^{+}\right)^{p^{t-1}}$ lies in the $G F(p)$-span of the block idempotents of $Z$, using [K91, (59)]. So $\left(z^{p^{t}}\right)^{p}=z^{p^{t}}$. However,

$$
\left(z^{p^{t}}\right)^{p}=\left(\tau^{+}\right)^{p^{t}} \neq\left(\tau^{+}\right)^{p^{t-1}}=z^{p^{t}}
$$

by the proof of Theorem 12. This contradiction shows that no such $z$ exists.

proof of Theorem 3. Let $g$ be a 2-regular element of $\mathcal{S}(n)$ and let $t$ be an involution which inverts $g$. If $X$ is a $\langle g\rangle$-orbit on $\{1, \ldots, n\}$, then so too is $X t$. So either $X$ is stabilized by $\langle t\rangle$, or $t$ contains the $|X|$-transpositions $\{(x, x t) \mid x \in X\}$ in its cycle decomposition. Suppose that $X$ is stabilized by $\langle t\rangle$. Then $t$ fixes some point, say $x_{0}$, in $X$, since $|X|$ is odd and $\langle t\rangle$ 
is a 2-group. It follows from the fact that $t$ inverts $g$ that $t$ contains the $(|X|-1) / 2$-transpositions $\left\{\left(x_{0} g^{j}, x_{0} g^{|X|-j}\right) \mid j=1, \ldots(|X|-1) / 2\right\}$ in its cycle decomposition.

Suppose that $g$ has $a_{i}$ orbits of size $i$, and that exactly $b_{i}$ of these are stabilized by $\langle t\rangle$. Then the number of transpositions in $t$ is

$$
\sum b_{i} \frac{(i-1)}{2}+\frac{\left(a_{i}-b_{i}\right)}{2} i=\sum \frac{a_{i} i-b_{i}}{2} .
$$

Moreover,

$$
\sum\left(a_{i} i-b_{i}\right) / 2 \leq \sum a_{i}(i-1) / 2,
$$

with equality if and only if $b_{i}=a_{i}$, for all $i$.

Given a set $\mathcal{X}$ of representatives for the orbits of $\langle g\rangle$ on $\{1, \ldots, n\}$, there is a unique involution $s$ which inverts $g$ and centralizes all members of $\mathcal{X}$. Let $\mathcal{T}$ be the conjugacy class of $\mathcal{S}(n)$ which contains $s$, and suppose that $t \in \mathcal{T}$ inverts $g$. By (20), the cycle decomposition of $s$, and hence $t$, consists of $\sum_{i} a_{i}(i-1) / 2$ transpositions. But then (21) implies that $t$ fixes an element from each $\langle g\rangle$-orbit. We deduce that

$$
\left|\left\{t \in \mathcal{T} \mid g^{t}=g^{-1}\right\}\right|:=\prod i^{a_{i}}
$$

equals the number of sets of representatives for the orbits of $\langle g\rangle$ on $\{1, \ldots, n\}$. A standard argument gives

$$
\left(\left(\mathcal{T}^{+}\right)^{2}, g\right)=\left|\left\{t \in \mathcal{T} \mid g^{t}=g^{-1}\right\}\right| 1_{F} .
$$

The theorem now follows from the fact that $\prod i^{a_{i}}$ is odd.

It remains to prove Proposition 4. First we need some notation for subgroups of $\mathcal{S}(n)$. Much of this is taken from [R93, 1.6].

Let $X$ and $Y$ be finite sets. We use $\mathcal{S}(X)$ to denote the group of all permutations of $X$. By convention all permutations act on the right. Let $H$ be a subgroup of $\mathcal{S}(X)$. If $h \in H$ and $y_{0} \in Y$, we can define a permutation $h\left(y_{0}\right)$ of $X \times Y$ via

$$
(x, y) h\left(y_{0}\right):=\left\{\begin{array}{ll}
(x h, y), & \text { if } y=y_{0}, \\
(x, y), & \text { if } y \neq y_{0},
\end{array} \quad \text { for all }(x, y) \in X \times Y .\right.
$$

The map $h \rightarrow h\left(y_{0}\right)$ gives an injection $H \hookrightarrow \mathcal{S}(X \times Y)$, whose image we denote by $H\left(y_{0}\right)$. We let $H^{Y}$ denote the group generated by $\left\{H\left(y_{0}\right) \mid y_{0} \in Y\right\}$. So $H^{Y}$ isomorphic to the external direct product of $|Y|$ copies of $H$.

Suppose that we have a collection of disjoint finite sets $\left\{X_{y} \mid y \in Y\right\}$ and groups $\left\{H_{y} \leq \mathcal{S}\left(X_{y}\right) \mid y \in Y\right\}$, indexed by the elements of $Y$. Then $\prod_{y \in Y} H_{y}$ 
denotes the group generated by $\left\{H_{y}(y) \mid y \in Y\right\}$. So $\prod_{y \in Y} H_{y}$ is an embedding of the external direct product of the groups $H_{y}$ in $\mathcal{S}\left(\cup_{y} X_{y}\right)$.

Let $K$ be a subgroup of $\mathcal{S}(Y)$. For $k \in K$, we can define a permutation $k^{*}$ of $X \times Y$ via

$$
(x, y) k^{*}:=(x, y k), \quad \text { for all }(x, y) \in X \times Y .
$$

The map $k \rightarrow k^{*}$ gives an injection $K \hookrightarrow \mathcal{S}(X \times Y)$, whose image we denote by $\Delta(K, X)$ (and $\Delta(K, n)$, if $|X|=n)$. In particular, $\Delta(K, X) \cong K$.

The wreath product $H$ Z $K$ of $H$ with $K$ is the subgroup of $\mathcal{S}(X \times Y)$ generated by $H^{Y}$ and $\Delta(K, X)$. A quick calculation shows that $h\left(y_{0}\right)^{k^{*}}=h\left(y_{0} k\right)$, for each $y_{0} \in Y, h \in H$ and $k \in K$. It follows that $H^{Y}$ is a normal subgroup of $H \backslash K$. We call $H^{Y}$ the base group of $H$ 乙 $K$. Also $H^{Y} \cap \Delta(K, X)=\{1\}$. So $H \imath K$ is isomorphic to a semi-direct product of $H^{Y}$ with $K$.

If $m$ is a positive integer, we will use $Z_{m}$ to denote the cyclic subgroup of $\mathcal{S}(m)$ generated by an $m$-cycle. The following is crucial to be proof of Proposition 4:

Proposition 22. Let $m$ and $n$ be positive integers with h.c.f. $(m, n)=1$. Then $Z_{m} \prec \mathcal{S}(n)=\Delta\left(Z_{m}, n\right) \times N$, for some group $N$.

Proof. Let $h$ be a generator of $Z_{m}$. A typical element of $Z_{m}<\mathcal{S}(n)$ is of the form $\prod_{i=1}^{n} h(i)^{\alpha_{i}} \sigma^{*}$, with $\sigma \in \mathcal{S}(n)$, and $0 \leq \alpha_{i} \leq m-1$, for $i=1, \ldots, n$. Define $\theta: Z_{m} \prec \mathcal{S}(n) \rightarrow Z_{m}$, by

$$
\theta\left(\prod_{i=1}^{n} h(i)^{\alpha_{i}} \sigma^{*}\right)=\prod_{i=1}^{n} h^{\alpha_{i}} .
$$

Then $\theta$ is a group homomorphism, since $h(i)^{\sigma^{*}}=h(i \sigma)$, for $i=1, \ldots, n$, and $\sigma \in \mathcal{S}(n)$.

Consider the generator $\delta:=\prod_{i=1}^{n} h(i)$ of $\Delta\left(Z_{m}, n\right)$. Since h.c.f. $(m, n)=1$, it follows that $\theta(\delta)=h^{n}$ is a generator of $Z_{m}$. So $\theta$ is onto, and $\operatorname{ker}(\theta) \cap$ $\Delta\left(Z_{m}, n\right)=\{1\}$. But $\Delta\left(Z_{m}, n\right)$ is central in $Z_{m} 2 \mathcal{S}(n)$. We conclude that

$$
Z_{m} \prec \mathcal{S}(n)=\Delta\left(Z_{m}, n\right) \times N, \quad \text { where } N=\operatorname{ker}(\theta) .
$$

Let

$$
\begin{aligned}
\operatorname{Fix}(H) & :=\{x \in X \mid x h=x, \text { for all } h \in H\}, \\
\operatorname{Mov}(H) & :=\{x \in X \mid x h \neq x, \text { for some } h \in H\} .
\end{aligned}
$$

Lemma 23. $\mathrm{C}_{\mathcal{S}(X)}(H)=\mathrm{C}_{\mathcal{S}(\operatorname{Mov}(H))}(H) \times \mathcal{S}(\operatorname{Fix}(H))$. If $\operatorname{Fix}(H)=\phi$ then $\mathbf{C}_{\mathcal{S}(X \times Y)}\left(H^{Y}\right)=\mathbf{C}_{\mathcal{S}(X)}(H)^{Y}$. 
Proof. Both statements are obvious.

Lemma 24. Suppose that $K$ acts transitively on $Y$. Then

$$
\mathrm{C}_{\mathcal{S}(X \times Y)}(\Delta(K, X))=\mathbf{C}_{\mathcal{S}(Y)}(K) \prec \mathcal{S}(X) .
$$

Proof. It is clear that $\mathbf{C}_{\mathcal{S}(Y)}(K) \prec \mathcal{S}(X) \subseteq \mathbf{C}_{\mathcal{S}(X \times Y)}(\Delta(K, X))$.

For each $x \in X$, the set $x \times Y:=\{(x, y) \mid y \in Y\}$ is a $\Delta(K, X)$-orbit on $X \times Y$. Moreover, each $\Delta(K, X)$-orbit equals $x \times Y$, for some $x \in X$.

Let $x \in X$ and $\sigma \in \mathbf{C}_{\mathcal{S}(X \times Y)}(\Delta(K, X))$. The previous paragraph implies that $(x \times Y) \sigma=x \sigma_{1} \times Y$, for some $\sigma_{1} \in \mathcal{S}(X)$. So for $y \in Y$ we have

$$
(x, y) \sigma=\left(x \sigma_{1}, y \sigma_{x}\right)
$$

where $\sigma_{x} \in \mathcal{S}(Y)$ depends on $x$. An easy calculation shows that $\sigma_{x} \in \mathbf{C}_{\mathcal{S}(Y)}(K)$. So $\sigma=\sigma_{1}^{*} \prod_{x \in X} \sigma_{x}$ lies in $\mathbf{C}_{\mathcal{S}(Y)}(K) \curlywedge \mathcal{S}(X)$. The lemma follows.

Corollary 25. Suppose that $H$ fixes no element of $X$ and that $K$ acts transitively on $Y$. Then $\mathbf{C}_{\mathcal{S}(X \times Y)}(H \curlywedge K)=\Delta\left(\mathbf{C}_{\mathcal{S}(X)}(H), Y\right)$.

Proof. We have

$$
\begin{aligned}
\mathbf{C}_{\mathcal{S}(X \times Y)}(H \succ K)= & \mathbf{C}_{\mathcal{S}(X \times Y)}\left(H^{Y}\right) \cap \mathbf{C}_{\mathcal{S}(X \times Y)}(\Delta(K, X)), \\
& \quad \text { using the definition of wreath product, } \\
= & \mathbf{C}_{\mathcal{S}(X)}(H)^{Y} \cap \mathbf{C}_{Y}(K) \prec \mathcal{S}(X), \quad \text { by Lemmas } 23 \text { and } 24, \\
= & \left\langle c\left(y_{0}\right) \mid c \in \mathbf{C}_{\mathcal{S}(X)}(H), y_{0} \in Y\right\rangle \cap \Delta(\mathcal{S}(X), Y) \\
= & \Delta\left(\mathbf{C}_{\mathcal{S}(X)}(H), Y\right) .
\end{aligned}
$$

Recall that $g$ is an element of $\mathcal{S}(n)$ and that $D$ is a Sylow $p$-subgroup of $\mathrm{C}=C_{\mathfrak{S}(n)}(g)$. We use the above results to compute $\mathbf{C}_{\mathcal{S}(n)}(D)$. Suppose that $g$ has $a_{i}$ cycles of length $i$ in its cycle decomposition, for $i=1,2, \ldots, n$.

Lemma 26. $\mathrm{C} \cong \prod_{i=1}^{n} Z_{i} \prec \mathcal{S}\left(a_{i}\right)$.

Proof. This is 4.1 .19 of [JK81].

If $n$ is an integer, write $n_{p}$ for the $p$-part of $n$ and $n_{p^{\prime}}$ for the $p$-regular part of $n$. So $n=n_{p} n_{p^{\prime}}$, and $n_{p}$ is a power of $p$, while $n_{p^{\prime}}$ is coprime to $p$. Let $a_{i}=\sum b_{i j} p^{j}$ be the base $p$-expansion of $a_{i}$, and let $P\left(a_{i}\right)$ be a Sylow $p$-subgroup of $\mathcal{S}\left(a_{i}\right)$. It is know that

$$
P\left(a_{i}\right) \cong \prod_{10} P\left(p^{j}\right)^{b_{i j}}
$$


where $P\left(p^{j}\right)$ is a Sylow $p$-subgroup of $\mathcal{S}\left(p^{j}\right)$. Here we restrict $j$ to those values for which $b_{i j} \neq 0$. Also $P\left(p^{j}\right)$ is a transitive subgroup of $\mathcal{S}\left(p^{j}\right)$, and the centre $\mathbf{Z}\left(P\left(p^{j}\right)\right)$ of $P\left(p^{j}\right)$ coincides with $\mathbf{C}_{\delta\left(p^{j}\right)}\left(P\left(p^{j}\right)\right)$.

See (9) in [O86] for another version of the following lemma:

Lemma 28. $D \cong \prod_{i_{p} \neq 1} \prod_{j}\left(\Delta\left(Z_{i_{p}}, i_{p^{\prime}}\right) \curlywedge P\left(p^{j}\right)\right)^{b_{i j}} \times \prod_{i_{p}=1} \Delta\left(P\left(a_{i}\right), i\right)$.

Proof. This follows from Lemma 26, (27), and the definition of the wreath product. Note that $\Delta\left(Z_{i_{p}}, i_{p^{\prime}}\right)$ is a Sylow $p$-subgroup of $Z_{i}$.

\section{Proposition 29.}

$$
\begin{aligned}
\mathbf{C}_{\mathcal{S}(n)}(D) \cong & \prod_{i_{p} \neq 1} \prod_{j} \Delta\left(Z_{i_{p}} \prec \mathcal{S}\left(i_{p^{\prime}}\right), p^{j}\right)^{b_{i j}} \\
& \times \prod_{i_{p}=1} \prod_{j>0}\left(\mathbf{Z}\left(P\left(p^{j}\right)\right) \imath \mathcal{S}(i)\right)^{b_{i j}} \times \mathcal{S}\left(\sum_{i_{p}=1} i b_{i 0}\right) .
\end{aligned}
$$

Proof. Suppose that $1 \leq i \leq n$ and $i_{p} \neq 1$. Then

$$
\begin{aligned}
& \mathbf{C}_{\mathcal{S}\left(i p^{j}\right)}\left(\Delta\left(Z_{i_{p}}, i_{p^{\prime}}\right) \curlywedge P\left(p^{j}\right)\right)=\Delta\left(\mathbf{C}_{\mathcal{S}(i)}\left(\Delta\left(Z_{i_{p}}, i_{p^{\prime}}\right)\right), p^{j}\right), \quad \text { by Corollary } 25 \\
& =\Delta\left(\mathbf{C}_{\mathcal{S}\left(i_{p}\right)}\left(Z_{i_{p}}\right) \prec \mathcal{S}\left(i_{p^{\prime}}\right), p^{j}\right), \quad \text { by Lemma } 24 \\
& =\Delta\left(Z_{i_{p}} \prec \mathcal{S}\left(i_{p^{\prime}}\right), p^{j}\right) \text {. }
\end{aligned}
$$
has no fixed points for $j>0$. Also

$$
\begin{aligned}
\mathbf{C}_{\mathcal{S}\left(i p^{j}\right)}\left(\Delta\left(P\left(p^{j}\right), i\right)\right) & =\mathbf{C}_{\mathcal{S}\left(p^{j}\right)}\left(P\left(p^{j}\right)\right) \curlywedge \mathcal{S}(i), \quad \text { by Lemma } 24 \\
& =\mathbf{Z}\left(P\left(p^{j}\right)\right) \curlywedge \mathcal{S}(i) .
\end{aligned}
$$

The proposition now follows from repeated applications of Lemma 23.

proof of Proposition 4. It follows from Propositions 22 and 29 that

$$
\mathbf{C}_{\mathcal{S}(n)}(D)=\prod_{i_{p} \neq 1} \prod_{j} \Delta\left(Z_{i_{p}}, i_{p^{\prime}} p^{j}\right)^{b_{i j}} \times M
$$

for some subgroup $M$ of $\mathcal{S}(n)$. Also, the projection of $g_{p}$ onto each factor $\Delta\left(Z_{i_{p}}, i_{p^{\prime}} p^{j}\right)$ generates that factor. The proposition now follows from standard properties of finite abelian groups.

\section{ACKnowledgement}

I am most grateful to Gordan James for pointing out how [M83, 1.9] might be used to prove Proposition 9. 


\section{REFERENCES}

[E90] M. Enguehard, Isométries parfaites entre blocs de groupes symétriques, S.M.F. Astérisque Vol. 181-182 (1990), p157-171.

[JK81] G. James, A. Kerber, The representation theory of the symmetric group, Encycl. of Math. and Applic. 16, Addison-Wesley Publ. Co., London, 1981.

[K91] B. Külshammer, Group-theoretical descriptions of ring-theoretical invariants of group algebras, Progress in Math. 95 (1991), 425-442.

[M83] G. E. Murphy, The Idempotents of the Symmetric Group and Nakayama's Conjecture, J. Algebra 81 (1983), 258-265.

[O80] J. B. Olsson, Lower Defect Groups, Comm. Alg. 8, No. 3 (1980), 261-288.

[O86] J. B. Olsson, Lower Defect Groups in Symmetric Groups, J. Algebra 104, 37-56 (1986).

[R93] D. J. S. Robinson, A course in the theory of groups, Graduate Texts in Math. 80, Springer-Verlag, New York, 1993.

E-mail address: jcmurray@eircom.net 